\title{
A new roadmap to improve translation of imaging biomarkers
}

\author{
Michael Skwarski ${ }^{1}$ and Geoff S Higgins ${ }^{*}, 1$ \\ ${ }^{1}$ Cancer Research UK/MRC Oxford Institute for Radiation Oncology, Department of Oncology, Old Road Campus Research \\ Building, Roosevelt Drive, Oxford OX3 7DQ, UK
}

Biomarkers are defined as 'characteristics that are objectively measured and evaluated as indicators of normal biological processes, pathological processes or pharmaceutical responses to a therapeutic intervention' (Biomarkers Definitions Working Group, 2001). Imaging biomarkers (IBs) constitute an important subclass of biomarker and their development represents an exciting and expanding field of oncology research.

Such biomarkers already have an important role in clinical practice in oncology: from screening and diagnosis, through to patient stratification, treatment planning, monitoring disease response and assessing treatment toxicity.

New IBs are used for research purposes to test in-vivo research hypotheses and are increasingly incorporated into early phase clinical trials testing novel anti-cancer treatments. For example, such biomarkers may identify the presence of treatment targets, confirm target modulation and monitor response. In drug development, they can act as gatekeepers in the drug development pipeline by providing crucial early data on treatment efficacy, accelerating the development of effective agents. Conversely, a lack of early response signal may halt development of ultimately ineffective treatments.

Despite an increase in new IBs, only a few have undergone full validation and qualification for routine research or clinical use. A key reason for this is a lack of effective, standardised, translational strategies. In response, Cancer Research UK (CRUK) and the European Organisation for Research and Treatment of Cancer (EORTC) assembled a panel of experts to consider these challenges, leading to publication of a consensus statement outlining a new 'Imaging Biomarker Roadmap for Cancer Studies' (O'Connor et al, 2016). This builds on previous US and European guidance (Dorfman et al, 2008; European Society of Radiology, 2013), and sets out a comprehensive strategy to improve future IB translation.

This proposed roadmap defines clear 'translational gaps', which must be crossed for IBs to become reliable measures suitable for research purposes and for clinical decision-making. In order to pass across these translational gaps, parallel defined processes must be undertaken consisting of: technical validation, biological and clinical validation, and assessment of cost effectiveness. To ensure effective navigation of this roadmap, a number of key recommendations have been made by this consensus group relating to these processes.

With regards to technical validation, this group calls for the accreditation of clinical centres and imaging laboratories to standards set by the IB community. Undeniably, accreditation will reduce variation in technical performance. However, the increasing rate of biomarker discovery, alongside continuous improvements in imaging equipment, software and data analysis, means that biomarker-specific site accreditation will inevitably lag behind new developments. It is therefore important that this additional regulation does not impede the development of novel IBs or hinder improvements to existing biomarkers. To further improve technical validation, it has also been recommended that repeatability (test-retest) and multicentre reproducibility studies should be conducted whenever possible. Although this is clearly important to evaluate the precision of IBs, such studies are costly, time consuming, and require significant additional funding and logistical support. To encourage such an approach, there must be widespread recognition of the importance of such data to this field of research and appropriate resources made available.

This new guidance also emphasises the need for extensive preclinical biological validation studies to ensure robust evaluation of imaging-biology correlation. It is then recommended that there is more transparency amongst researchers when reporting results. Furthermore, it is suggested that 'imaging repositories' are created with the aim of accelerating biological validation by pooling relevant data. It is important that when combining multicentre data in this way, evidence of technical validation is adequately scrutinised and concerns surrounding intellectual property are addressed. This approach does, however, provide the opportunity for rapid biological validation and may potentially require the use of fewer animals or patient participants in individual studies.

*Correspondence: Dr GS Higgins; E-mail: geoffrey.higgins@oncology.ox.ac.uk 
This imaging biomarker roadmap highlights the specific and varied challenges in developing IBs. An important underlying theme to many of the recommendations made is that there is a need to create a more open and collaborative IB research environment. By ensuring standardisation of data acquisition and analysis, accredited technical validation and the transparent sharing of biological and clinical validation data, it is hoped that more IBs will cross translational gaps and become useful research and clinical decision-making tools of the future.

\section{CONFLICT OF INTEREST}

The authors declare no conflict of interest.

\section{REFERENCES}

Biomarkers Definitions Working Group (2001) Biomarkers and surrogate endpoints: preferred definitions and conceptual framework. Clin Pharmacol Ther 69(3): 89-95.
Dorfman GS, Sullivan DC, Schnall MD, Matrisian LM. Translational Research Working Group (2008) The Translational Research Working Group developmental pathway for image-based assessment modalities. Clin Cancer Res 14(18): 5678-5684.

European Society of Radiology (2013) ESR statement on the stepwise development of imaging biomarkers. Insights Imaging 4(2): $147-152$.

O'Connor JP, Aboagye EO, Adams JE, Aerts HJ, Barrington SF, Beer AJ, Boellaard R, Bohndiek SE, Brady M, Brown G, Buckley DL, Chenevert TL, Clarke LP, Collette S, Cook GJ, deSouza NM, Dickson JC, Dive C, Evelhoch JL, Faivre-Finn C, Gallagher FA, Gilbert FJ, Gillies RJ, Goh V, Griffiths JR, Groves AM, Halligan S, Harris AL, Hawkes DJ, Hoekstra OS, Huang EP, Hutton BF, Jackson EF, Jayson GC, Jones A, Koh DM, Lacombe D, Lambin P, Lassau N, Leach MO, Lee TY, Leen EL, Lewis JS, Liu Y, Lythgoe MF, Manoharan P, Maxwell RJ, Miles KA, Morgan B, Morris S, Ng T, Padhani AR, Parker GJ, Partridge M, Pathak AP, Peet AC, Punwani S, Reynolds AR, Robinson SP, Shankar LK, Sharma RA, Soloviev D, Stroobants S, Sullivan DC, Taylor SA, Tofts PS, Tozer GM, van Herk M, Walker-Samuel S, Wason J, Williams KJ, Workman P, Yankeelov TE, Brindle KM, McShane LM, Jackson A, Waterton JC (2016) Imaging biomarker roadmap for cancer studies. Nat Rev Clin Oncol; e-pub ahead of print 11 October 2016; doi:10.1038/ nrclinonc.2016.162. 